\title{
Aprendizaje activo para el desarrollo de la psicomotricidad y el trabajo en equipo
}

\author{
Active Learning to Develop Motor Skills and Teamwork
}

\section{Aprendizagem ativa para desenvolver habilidades motoras e trabalho em equipe}

\author{
Johanna Lorena Aristizabal-Almanza' \\ Tecnológico de Monterrey \\ Bogotá, Colombia \\ loremaster6@hotmail.com \\ D http://orcid.org/0000-0001-9658-2532 \\ Arcelia Ramos-Monobe ${ }^{2}$ \\ Tecnológico de Monterrey \\ Guadalajara, México \\ arcelia.ramos.monobe@itesm.mx \\ iD http://orcid.org/0000-0001-8052-7272 \\ Violeta Chirino-Barceló \\ Tecnológico de Monterrey \\ Ciudad de México, México \\ vchirino@itesm.mx \\ iD http://orcid.org/0000-0003-0106-2708
}

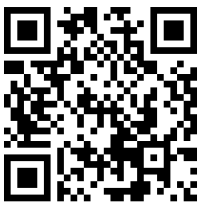

Recibido • Received • Recebido: 04 / 07/ 2016

Corregido • Revised • Revisado: 13 / 11 / 2017

Aceptado • Accepted • Aprovado: 14/ 12 / 2017

\begin{abstract}
'Administradora Deportiva de la Universidad Distrital de Colombia y Magister en Educación con Énfasis en Procesos de Enseñanza Aprendizaje del Tecnológico de Monterrey en México. Actualmente, docente de expresión corporal, con niñez de básica y secundaria, con experiencia principalmente, alrededor del campo de la educación física, específicamente en el área de la expresión corporal, danzas, recreación y deporte desde hace ocho años. Asimismo, ha participado en iniciativas que involucran la planeación, organización y ejecución de eventos deportivos y artísticos, y la promoción del deporte y el aprovechamiento del tiempo libre a través de escuelas de formación.

${ }^{2}$ Licenciada en Psicología por la Universidad Autónoma de Guadalajara. Maestra en Educación con especialidad en Desarrollo Cognitivo por el Tecnológico de Monterrey, desde 1986 participa como docente y especialista en la formación de docentes en la institución. Ha colaborado como tutora de los cursos de la maestría en educación; particularmente en proyectos de investigación desde 2004, las investigaciones giran alrededor de intervenciones pedagógicas asociadas al aprendizaje activo.

${ }^{3}$ Doctora en Innovación y Tecnología Educativa por el Tecnológico de Monterrey. Profesora titular en la Escuela de Educación Humanidades y Ciencias Sociales del ITESM, con líneas de investigación: Aprendizaje activo y aprendizaje móvil con publicaciones en libros y revistas arbitradas. Moderó en 2012 para OEA el Foro Virtual sobre TIC y Educación y fue relatora en el Foro de la Sociedad Civil del Continente Americano en Washington DC, para el Summit de las Américas.
\end{abstract}


doi: http://dx.doi.org/10.15359/ree.22-1.16

URL: http://www.una.ac.cr/educare

CORREO: educare@una.cr

Resumen: Se presentan los resultados de un proceso de investigación-acción, con datos cuantitativos y cualitativos, cuyo objetivo fue establecer cómo el uso de los principios del aprendizaje activo en la práctica docente, particularmente la colaboración, incidía en los procesos de aprendizaje, en específico, en el trabajo en equipo y el desempeño psicomotriz de un grupo de estudiantes de primer grado. Participaron 20 estudiantes que se seleccionaron mediante muestreo intencional basado en criterios, de grado primero de nivel básico de un colegio privado en Bogotá, Colombia. Se aplicaron técnicas como la observación, se realizaron entrevistas y recolectaron datos con base en pruebas de motricidad estandarizadas, antes y después del proceso; el análisis de datos se realizó mediante triangulación de los datos cualitativos, y el análisis comparativo del perfil inicial y final del estudiantado para los cuantitativos. Los resultados indican que luego de la intervención didáctica en la cual se utilizó colaboración fundamentada en aprendizaje activo, el estudiantado logró una variación positiva en el desempeño de las competencias observadas; que ser parte de un equipo de trabajo incidió positivamente en el logro de los objetivos propuestos; que al realizar, de manera constante, reflexión individual sobre sus prácticas, fueron capaces de identificar aciertos y desaciertos para construir conocimiento en la interacción con las demás personas; que se facilitó el desarrollo de saberes en conexión de los aprendizajes previos y se logró que el estudiantado fuera más responsable y autodirigido. Asimismo, se reconoció la importancia de innovar en los procesos de enseñanza-aprendizaje en el aula, de manera que se cuente con datos significativos para el personal docente e investigador que opte por proveer experiencias de aprendizaje basadas en principios de aprendizaje activo.

Palabras claves: Aprendizaje activo; psicomotricidad; trabajo en equipo; investigación-acción.

Abstract: This action-research project was conducted to determine how the use of principles of active learning, specifically collaboration, had an effect on psychomotor performance and achievement in teamwork. The research setting included 20 students of first grade from a private school located in Bogota, Colombia. The students were selected through not randomized sampling based on criteria. The methodological process included observation, interviews, and a scale based on standardized tests to measure skills; the latter was applied before and after the intervention. Data analysis was performed using a triangulation of qualitative data, and through comparative analysis of the initial and final student profile for quantitative inputs. The results showed that, after the intervention with collaborative techniques based on action learning, students achieved a positive variation in their performance. Being part of a team positively affected the achievement of the objectives. Systematical reflection on their practices fostered their capacity to identify strengths and weaknesses to build knowledge in interaction with others. Knowledge construction was nurtured based in their previous experiences. Students showed more accountability and self-directed learning behaviors, according to their age. Overall the experience showed the importance of research and innovation in the classroom in order to provide meaningful data, so teachers and researchers can engage in providing learning experiences based in active learning.

Keywords: Action learning; psychomotor activity; teamwork; action research.

Resumo: São apresentados os resultados de um processo de investigação-ação, com dados quantitativos e qualitativos, cujo objetivo foi estabelecer como o uso dos princípios da aprendizagem ativa no ensino, particularmente a colaboração, impacta nos processos de aprendizagem, especificamente no trabalho em equipe e desempenho psicomotor de um grupo de estudantes da primeira série. Participaram 20 estudantes, que foram selecionados por amostragem intencional, com base em critérios de nível básico da 


\begin{abstract}
primeira série do ensino fundamental de uma escola particular em Bogotá, Colômbia. As técnicas aplicadas foram a observação, entrevistas e também foram recolhidos dados com base em testes padronizados de coordenação motora, antes e após o processo; a análise dos dados foi realizada utilizando triangulação de dados qualitativos. Com os dados quantitativos foram feitos análise comparativa dos perfis iniciais e finais de estudantes. Os resultados indicam que, após a intervenção educativa na qual a colaboração com base na aprendizagem ativa é usada, os estudantes alcançaram uma variação positiva no desempenho das competências observadas; o fato de fazer parte de uma equipe de trabalho contribuiu positivamente na realização dos objetivos propostos; ao realizar, constantemente, a reflexão individual sobre as suas práticas, foram capazes de identificar os erros e os acertos na construção de conhecimento interatuando com os demais; que facilitou o desenvolvimento do conhecimento relacionado com a aprendizagem prévia e ajudou em que os estudantes fossem mais responsáveis e autodirigidos. Além disso, se reconhece a importância da inovação nos processos de ensino-aprendizagem na sala de aula, com a finalidade de contar com dados significativos para docente e pesquisadores que decidam por investigar experiências fundamentadas em princípios de aprendizagem ativa.
\end{abstract}

Palavras-chave: aprendizagem ativa, habilidades motoras, trabalho em equipe, investigação-ação.

\title{
Introducción
}

Las didácticas aplicadas en el aula facilitan el aprendizaje del estudiantado cuando estos son agentes activos del proceso educativo (Huber, 2008). Al investigar la eficacia de la colaboración como didáctica de una intervención pedagógica que integra los principios de aprendizaje activo, desde un paradigma pragmático (Carr y Kemmis, 1988), se puede vincular el beneficio en la adopción de prácticas de aprendizaje que enfocan la autorregulación y"aprender a aprender" del estudiantado con la mejora de la práctica docente. Por tanto, el aprendizaje activo constituye una metodología educativa que centra al estudiantado como agente principal, a partir de la interacción con las demás personas; que construye conocimiento desde su propia reflexión y vivencias situadas en un contexto determinado, donde cada docente es un agente facilitador y guía, en busca de la formación integral del individuo.

Touriñan (2011) concibe la intervención pedagógica como una acción intencional en la práctica educativa, respecto a los propósitos y métodos desarrollados en el conocimiento de la educación y la actividad del sistema educativo. En el ámbito de la educación física, el desarrollo de la expresión corporal, entendida como la educación por medio del movimiento (Gómez, 2002), enfoca el desarrollo motriz del estudiantado, mediante la mejora paulatina de sus habilidades coordinativas, de lateralidad y control corporal; al igual que el desarrollo de las habilidades de interacción social de trabajo en equipo (Hernández y Anello, 1998), a partir de la apropiación de las características de interdependencia positiva. Todo ello permite alcanzar más fácilmente los objetivos de aprendizaje cuando son parte de un equipo colaborativo, por lo tanto, resulta un mecanismo efectivo para maximizar su propio aprendizaje y el de los demás individuos. 
doi: http://dx.doi.org/10.15359/ree.22-1.16

URL: http://www.una.ac.cr/educare

CORREO: educare@una.cr

Las intervenciones didácticas en educación física tienen la particularidad de integrar elementos humanos, físicos, y cognitivos en procesos estructurados por un personal docente que considere el perfil de sus estudiantes y promueva el desarrollo de las competencias planteadas con un enfoque integral; buscan potenciar el desarrollo de habilidades individuales y sociales, a través de una definición clara y planeada en los procesos y su evaluación (Osorio y López, 2014).

Blanco (2006) enfatiza la importancia de priorizar en las tareas que desarrollencompetencias que no solo contemplen las habilidades cognitivas (saberes), sino también las aptitudes (saber hacer), las actitudes (querer hacer) y valores (ser), por lo que la expresión corporal busca la formación integral del estudiantado. Vaca (2007) determina que la operacionalización de la expresión corporal radica en el perfeccionamiento de habilidades motoras que favorezcan el proceso de exploración del ambiente; los conceptos de lateralidad, dirección, velocidad, locomoción y percepción sensorial, junto con la mezcla de procesos mentales como el observar, descubrir, comparar, analizar, escuchar, evaluar o seguir instrucciones, que constituyen un verdadero significado global de la enseñanza de la corporeidad. De manera tal que en esta investigación se buscó determinar los efectos de la práctica intencional de los principios de aprendizaje activo y la colaboración, en los aprendizajes específicos del estudiantado, particularmente su desempeño motriz y el trabajo en equipo.

\section{Estado de la cuestión}

El estudiantado puede alcanzar más fácilmente los objetivos de aprendizaje cuando es parte de un equipo colaborativo. La investigación de Torrelles et al. (2011) plantea que para que se dé el principio de colaboración, el estudiantado debe tener claros los objetivos de aprendizaje y las habilidades de cada integrante del equipo con el fin de generar una interdependencia positiva como base del trabajo colaborativo.

Por su parte, Gil, Gutiérrez y Madrid (2013), en su investigación cualitativa, plantean la expresión corporal desde el concepto de la danza como enseñanza kinestésica, que permite desarrollar competencias de tipo social, para la interacción con los demás seres desde la mirada de la sociedad actual. Para Gil et al. (2013), el aprender a desarrollar las habilidades sociales resulta fundamental en la formación del estudiantado, en la búsqueda de mantener buenas relaciones con personas en los diferentes contextos de su vida. Los resultados más importantes de esta investigación se relacionan a la efectividad de la danza como estrategia didáctica de la expresión corporal en el fomento de competencias sociales.

Se puede reafirmar la importancia de la investigación para la innovación en el aula desde la implementación de didácticas y metodologías que empoderen al estudiantado en el proceso de aprendizaje, con el fin de prepararlo para desempeñarse en el futuro.

\section{4} Johanna Lorena Aristizabal-Almanza, Arcelia Ramos-Monobe y Violeta Chirino-Barceló

Los artículos de la Revista Electrónica Educare del Centro de Investigación y Docencia en Educación de la Universidad Nacional, Costa Rica, se comparten bajo términos de la Licencia Creative Commons: Reconocimiento, № Comercial, Sin Obra Derivada 3.0 Costa Rica. Las autorizaciones adicionales a las aquí delimitadas se pueden obtener en el correo: educare@una.cr 
De acuerdo con DaFonseca (2000), en la etapa de infantes entre seisy siete años, el desarrollo psicomotriz debe corresponder a evidencias de tipo perceptivo-motriz donde el estudiantado muestre habilidades de disociación, direccionalidad, auto-identificación y localización corporal, a través del dominio de sus habilidades básica en la coordinación, orientación espaciotemporal y control corporal.

Domingo (2008) indica que, en la etapa de infantes, se debe empezar a interactuar con los demás y con el entorno a través del desarrollo de habilidades comunicativas, de escucha y de responsabilidad individual para la contribución al alcance de un objetivo común. Sin embargo, estudiantes de grado primero de escuela elemental en un colegio particular en Colombia evidenciaban limitaciones para desarrollar movimientos coordinativos simultáneos, al igual que en el control y percepción de movimientos, también en la capacidad de interacción con sus compañeros y compañeras y el medio que les rodea.

Esta investigación pretendió explorar la relación de la expresión corporal y el trabajo en equipo como dos finalidades formativas, vinculadas a una intervención con la colaboración basada en aprendizaje activo. Planteando la siguiente pregunta de investigación: ¿Cómo incide la colaboración basada en principios de aprendizaje activo, en las variaciones observadas en el desempeño psicomotriz del estudiantado para el desarrollo de su expresión corporal y en los niveles de logro de la competencia genérica de trabajo en equipo, en estudiantes pertenecientes al grado primero del colegio Jordán de Sajonia?

\section{Marco teórico}

El aprendizaje activo es una aproximación metodológica centrada en el estudiantado, con la premisa de que el conocimiento se construye a partir de la interacción con los demás individuos, apoyándose en la reflexión y vivencias situadas en un contexto determinado (Howell en Koo, 1999; Schwartz y Pollishuke, 1998; Silberman, 2005), que busca desarrollar la capacidad de pensamiento crítico (Revans, 1983).

El personal docente es un agente facilitador y guía que busca la formación integral del individuo, estructurando actividades específicas de enseñanza que permitan una relación directa entre la práctica y la teoría buscando la trascendencia y aplicación de los saberes a diferentes contextos y situaciones de su vida (Huber, 2008). Para ello realiza un proceso reflexivo que visualiza su aplicación en etapas: planificación, contextualización, reflexión individual, acción / práctica, reflexión colectiva, evaluación y mejora continua (Chirino, Ramos y Lozano, 2015; Huber, 2008). Revans (1983) menciona la importancia de reflexionar en estrategias pedagógicas que faciliten el aprendizaje desde la movilización del estudiantado hacia la reflexión, liderazgo y creatividad, considerando el aprendizaje entre pares. 
doi: http://dx.doi.org/10.15359/ree.22-1.16

URL: http://www.una.ac.cr/educare

CORREO: educare@una.cr

\section{El trabajo colaborativo aplicado desde principios de aprendizaje activo}

En el aprendizaje activo, el trabajo colaborativo es la vía para que el alumnado aprenda a interactuar con los demás seres, mediante un esfuerzo conjunto que tiene como fin la transformación de una realidad o la solución de un problema (Revans, 1983). El trabajo colaborativo, en su connotación de competencia genérica, se define como un proceso grupal en el que se observa la participación activa, reflexiva y propositiva de cada individuo aportando sus capacidades, conocimientos y habilidades en pro de la consecución de un bien común (Villa y Poblete, 2007); que permite maximizar el propio aprendizaje y el de las demás personas y que requiere operar con la definición de objetivos comunes claros, donde el conocimiento mutuo sobre las habilidades de cada miembro del equipo permiten generar una interdependencia positiva (Johnson, Johnson y Holubec, 1999). Asimismo, implica el establecimiento de responsabilidades individuales que integra elementos de evaluación y realimentación del desempeño intragrupal para la mejora (Domingo, 2008).

Domingo (2008) indica que el trabajo colaborativo necesita tanto de competencias disciplinares específicas y conocimientos, como de competencias interpersonales para determinar la buena relación con los demás individuos, como la motivación intrínseca, la comunicación asertiva, el liderazgo, la toma de decisiones y el manejo de conflictos, entre otras.

Johnson et al. (1999) establecen que los equipos que se integran en procesos de aprendizaje colaborativo tienen un sentido de pertenencia grupal que se manifiesta en que todos saldrán con una ganancia o fracaso derivada de la calidad de su cooperación, del trabajo mancomunado y de la interdependencia positiva.

\section{Aprendizaje activo y colaborativo para el desarrollo de la expresión corporal a partir del desempeño psicomotriz en educación básica}

La educación física busca potenciar el desarrollo de habilidades individuales y sociales, a través de una definición clara y planeada en sus procesos. Blanco (2006) enfatiza la importancia de priorizar en las tareas que desarrollen competencias que no solo contemplen las habilidades cognitivas (saberes), sino también las aptitudes (saber hacer), las actitudes (querer hacer) y valores (ser), por lo que la expresión corporal busca la formación integral del estudiantado. Bolaños (2006) plantea que es importante considerar las actividades de educación de movimiento en cuanto a la interacción continua del sujeto con el ambiente y con las demás personas. Así, pues, el término de psicomotricidad, integra las habilidades cognitivas, emocionales, simbólicas y sensorio-motrices, expresadas a través de su interacción dentro de un contexto social con el fin de potenciar el desarrollo personal e integral del individuo (García, 2009).

La expresión corporal, por su parte, incorpora todo lo relacionado con el conocimiento y manejo del propio cuerpo en acciones relacionadas con movimiento, locomoción, cualidades físicas, coordinación, desplazamientos, entre otras (Gómez, 2002). Puede entenderse, desde un enfoque

\footnotetext{
6 Johanna Lorena Aristizabal-Almanza, Arcelia Ramos-Monobe y Violeta Chirino-Barceló
}

Los artículos de la Revista Electrónica Educare del Centro de Investigación y Docencia en Educación de la Universidad Nacional, Costa Rica, se comparten bajo términos de la Licencia Creative Commons: Reconocimiento, № Comercial, Sin Obra Derivada 3.0 Costa Rica. Las autorizaciones adicionales a las aquí delimitadas se pueden obtener en el correo: educare@una.cr 
simple, cómo el movimiento, entendido a partir del desplazamiento que un cuerpo tiene por el espacio (Bolaños, 2006), hasta uno más integral, como la concepción en la que para el niño o la niña, el movimiento constituye su principal fuente de vitalidad y aprendizaje (Gómez, 2002) y puede ser medida en la realización de todo tipo de movimientos que le permiten al estudiantado reforzar su percepción de imagen corporal en la interacción con el entorno y diferentes situaciones (García, 2009).

Vaca (2007) determina que la operacionalización de la expresión corporal, que constituye un verdadero significado global de la enseñanza de la corporeidad, radica en el perfeccionamiento de habilidades motoras que favorezcan el proceso de exploración del ambiente, los conceptos de lateralidad, dirección, velocidad, locomoción y percepción sensorial, junto con la mezcla de procesos mentales como observar, descubrir, comparar, analizar, escuchar, evaluar o seguir instrucciones. Esto lleva a identificar que es necesario incentivar conductas reflexivas y habilidades motoras generales y específicas que constituyen la integralidad en la formación del estudiantado, desde lo biológico, social, emocional e intelectual, suministrando experiencias de aprendizaje significativas en su interacción social. Concluye así la importancia de incidir en este proceso durante una intervención didáctica.

\section{Metodología}

Este estudio fue realizado con un enfoque de investigación-acción (Elliott, 1993; Lewin, 1988), mediante una intervención didáctica, que corre paralela a las etapas del proceso de investigación. En su diseño se establecieron las etapas de implementación de la intervención, la evaluación diagnóstica, la recolección de datos, la evaluación final y la obtención de la percepción del estudiantado respecto a la experiencia vivida. Las etapas de la investigación se muestran en la Figura 1.

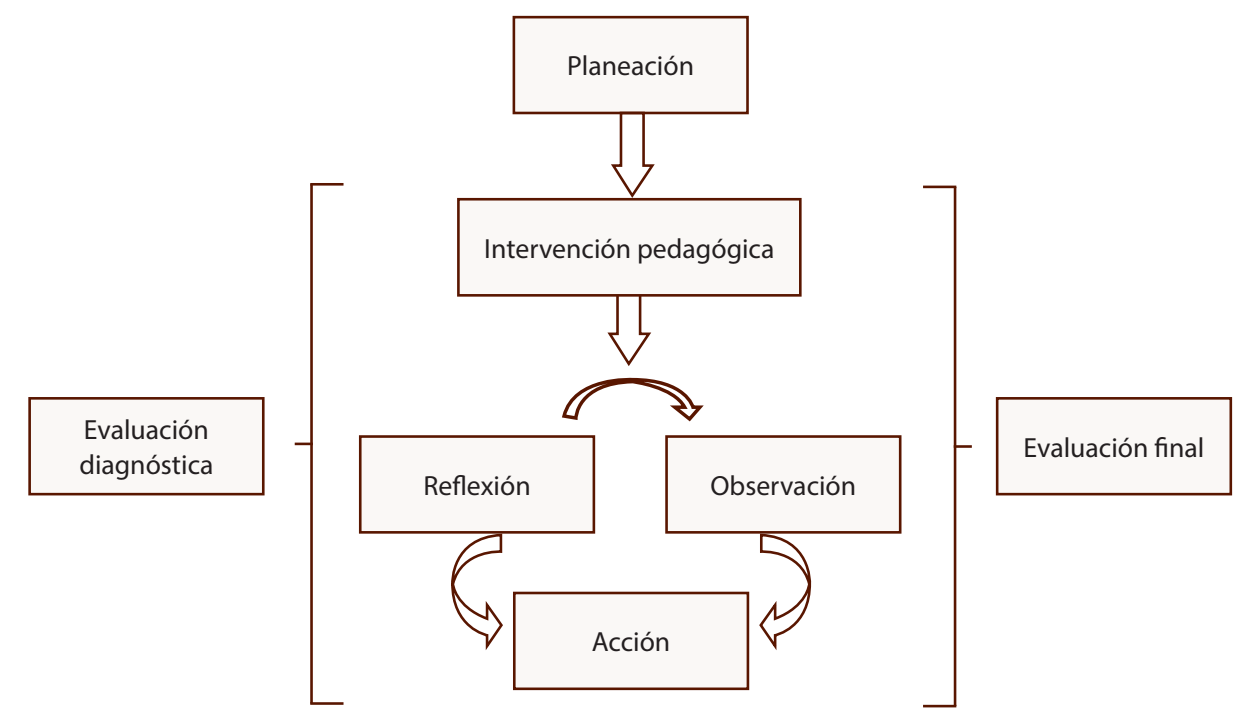

Figura 1: Etapas de la investigación basada en Carr y Kemmis (1988). 
doi: http://dx.doi.org/10.15359/ree.22-1.16

URL: http://www.una.ac.cr/educare

CORREO: educare@una.cr

La investigación se realizó en un colegio privado, ubicado en Bogotá, Colombia, con 20 estudiantes cuya selección se llevó a cabo mediante muestreo por conveniencia con los criterios de accesibilidad y proximidad de los sujetos (Valenzuela y Flores, 2012). Se seleccionaron como unidades de análisis las habilidades psicomotrices y la competencia de trabajo en equipo como objeto de esta investigación.

La recolección de datos implicó el uso de la observación como técnica durante el proceso de la intervención, se utilizó una guía para las seis sesiones de clase, incluía preguntas orientadoras y reflexivas; se enfocó en los elementos del trabajo en equipo como: la claridad en los objetivos de aprendizaje, las habilidades de cada participante del equipo desde la característica de interdependencia positiva y el desempeño de los equipos en la realización de tareas específicas, creadas a partir de los constructos teóricos propuestos por Johnson et al. (1999). Asimismo, como parte del proceso reflexivo de la investigación acción se validó, mediante la observación, si las estrategias pedagógicas planteadas para la intervención facilitaron el aprendizaje desde la movilización del estudiantado hacia la reflexión, liderazgo y creatividad, considerando el aprendizaje entre pares, como característica principal del aprendizaje activo (Huber, 2008).

Además, se aplicó una escala de estimación a manera de autorreporte para definir el desempeño del estudiantado durante la intervención pedagógica. Esta se ajusta a los procesos de reflexión individual y colectiva de un proceso de aprendizaje activo (Chirino et al., 2015). La escala se construyó a partir de afirmaciones que reflejaban el desempeño esperado tanto en el trabajo en equipo (Johnson et al., 1999) como en su desempeño psicomotriz, acorde a los resultados esperados de la intervención. Tanto en la guía de observación como en la escala de estimación, los ítems fueron categorizados a priori (Cisterna, 2005).

En cuanto a la psicomotricidad, se aplicó el instrumento de evaluación de habilidades psicomotrices-de elaboración propia a partir del compendio de los instrumentos estandarizados como el Test de coordinación de Ozeretski (1988), el Test de control corporal de Berges y Lézine (1975) y el Test de orientación latero-espacial (Galifret-Granjon, 1984). Se partió del protocolo establecido por los autores, conjuntando de una batería múltiple de aptitudes para las edades específicas de los sujetos participantes-. Se realizaron dos aplicaciones, diagnóstica y final; se contrastaron los resultados con objetivo de valorar el avance del estudiantado posterior a la intervención pedagógica. Finalmente, se realizó una entrevista grupal semi-estructurada, en formato de focus group a un grupo seleccionado de cinco alumnos y alumnas de la muestra.

La intervención implicó el diseño e implementación de una secuencia didáctica (Apéndice A) donde se observó la autorreflexión, participación, colaboración y motivación; como indicadores del aprendizaje activo (Chirino et al., 2015); durante la implementación se recolectaron los datos. La intervención constó de seis sesiones de cuarenta y cinco minutos cada una. Durante esta se siguió el proceso de investigación acción, observando y reflexionando.

8 Johanna Lorena Aristizabal-Almanza, Arcelia Ramos-Monobe y Violeta Chirino-Barceló

Los artículos de la Revista Electrónica Educare del Centro de Investigación y Docencia en Educación de la Universidad Nacional, Costa Rica, se comparten bajo términos de la Licencia Creative Commons: Reconocimiento, № Comercial, Sin Obra Derivada 3.0 Costa Rica. Las autorizaciones adicionales a las aquí delimitadas se pueden obtener en el correo: educare@una.cr 
Para el análisis de resultados se recurrió a la triangulación de los datos obtenidos, a fin de dar respuesta a la pregunta de investigación y concluir dando sentido a la información y los hallazgos observados. Las categorías de resultados se establecieron a priori (Cisterna, 2005), con base en la pregunta de investigación. Los datos recolectados a través de la guía de observación, la escala de estimación y la entrevista grupal se clasificaron de acuerdo con las categorías preestablecidas y se contrastaron conceptualmente y con los datos recolectados. Se realizó una comparación del perfil inicial y final de los participantes del test psicomotriz y de la escala de valoración que nos permitió evaluar la competencia de trabajo en equipo.

\section{Resultados}

La intervención pedagógica se desarrolló conforme lo planeado durante el año lectivo 2015-2016. Los datos que sustentan sus resultados se presentan de acuerdo con las variables de la investigación: psicomotricidad, trabajo en equipo y aprendizaje activo. No obstante, aluden al proceso de la intervención: a) un primer momento de diagnóstico donde se evaluó el estado inicial en cuanto a psicomotricidad y la competencia de trabajo en equipo de participantes; b) el proceso de la intervención en la que se obtienen los datos que revelan el aprendizaje activo experimentado por el estudiantado y; c) el momento de cierre y evaluación final del desempeño psicomotriz y de trabajo en equipo a fin de determinar las variaciones en el desempeño durante la intervención.

La psicomotricidad comprende los movimientos del cuerpo que permiten al alumnado la apropiación de conceptos asociados a la lateralidad, locomoción, control corporal y otros procesos mentales involucrados como el seguimiento de instrucciones, la observación y la escucha (Vaca, 2007). En esta investigación la evaluación de la psicomotricidad se centró en coordinación, control corporal y orientación latero-espacial. Los resultados se describen a continuación: se presentan, de forma ordenada, el diagnóstico y la evaluación final, para concluir con la comparación.

Diagnóstico: Coordinación. Todos los niños y niñas presentaron dificultades en sus movimientos simultáneos al realizar los ejercicios prepropuestos, que consideran las funciones coordinativas viso-manual y viso-pédica (ver figura 2). El diagnóstico implicó determinar en cuál intento el estudiantado logró ejecutar el ejercicio y la cantidad de fallos en el proceso. En la coordinación viso-manual el $80 \%$ logró realizar el ejercicio hasta el segundo intento, con un promedio de 11 a 15 faltas. En la coordinación viso-pédica se observó que el 60\% logró ejecutar el ejercicio hasta el segundo intento con un promedio de faltas de 6 a 10. Lo esperado de acuerdo con la teoría (Ozeretski, 1988) era un máximo de 0 a 5 faltas en su ejecución. 
doi: http://dx.doi.org/10.15359/ree.22-1.16

URL: http://www.una.ac.cr/educare

CORREO: educare@una.cr
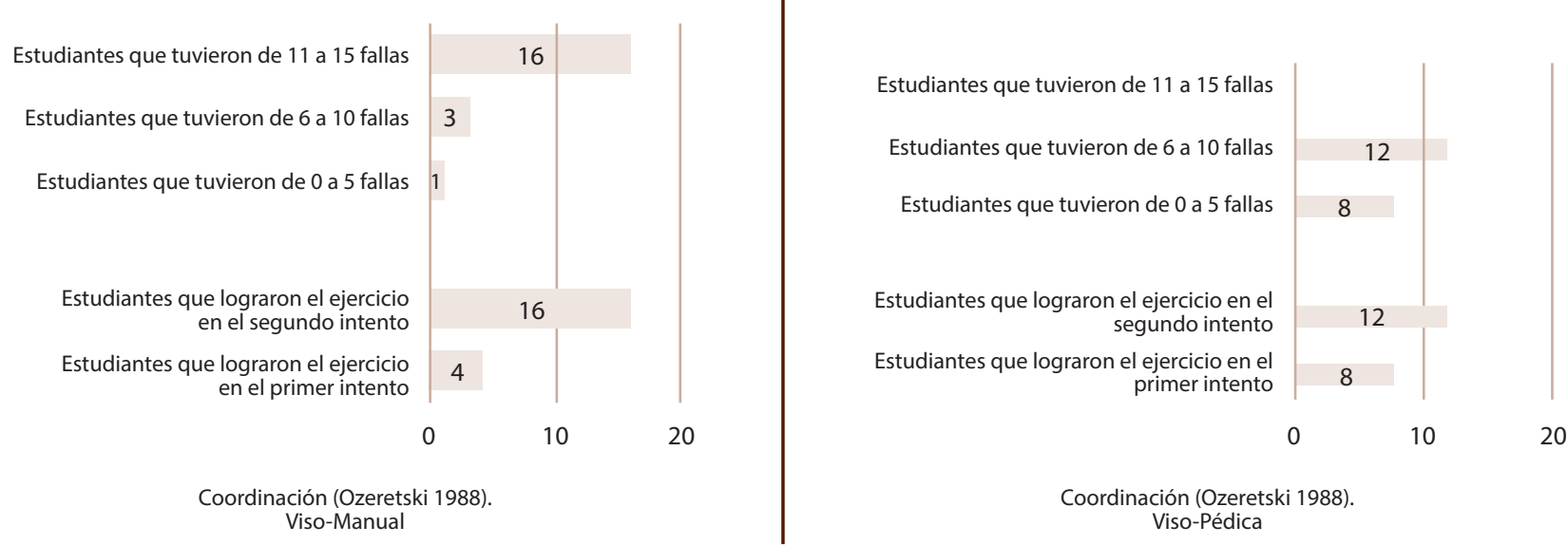

Figura 2: Resultados del Pretest de coordinación.

Diagnóstico: Control corporal. En la evaluación diagnóstica, el 75\% de estudiantes mostró un desempeño de medio a bajo con respecto a su edad y el desarrollo motor (Ver figura 3), ya que no lograron imitar los movimientos propuestos con una precisión adecuada de sus dimensiones corporales, dificultándoseles realizar movimientos controlados y precisos, lo cual es conforme con la teoría (Berges y Lézine, 1975), pues se espera que el estudiantado pueda realizar al menos 9 de los 10 movimientos establecidos en el test con dominio corporal y estabilidad en la ejecución; en este caso solo 5 estudiantes lo lograron.

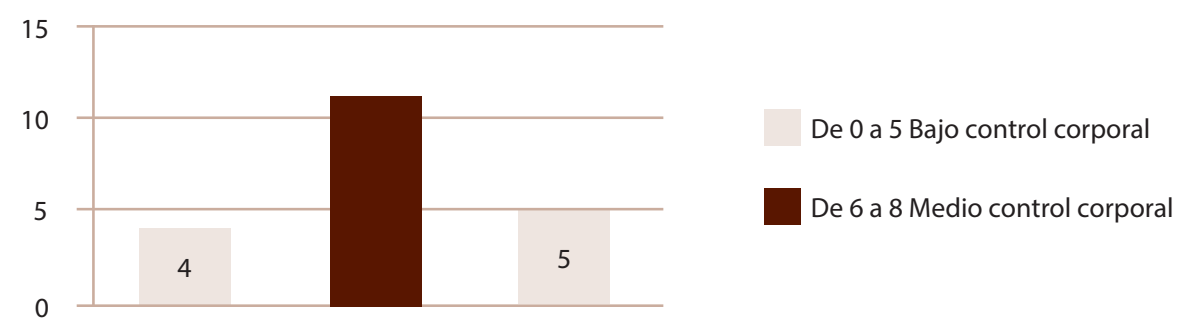

Control corporal (Berges y Lezine, 1975).

Puntos logrados por el estudiantado en cada imitación: Diagnóstico.

Figura 3: Resultados del Pretest de control corporal.

Diagnóstico: Lateralidad. Solo el $25 \%$ de los niños y niñas resolvieron correctamente situaciones de lateralidad, mientras que el $75 \%$ restante obtuvo una puntuación entre 0 y 4, menor que el mínimo definido en la teoría (Galifret-Granjon, 1984) para que el test sea considerado bien resuelto, según la edad correspondiente, como se muestra en la Figura 4. Los datos se presentan agrupados por la frecuencia con respecto al logro. 


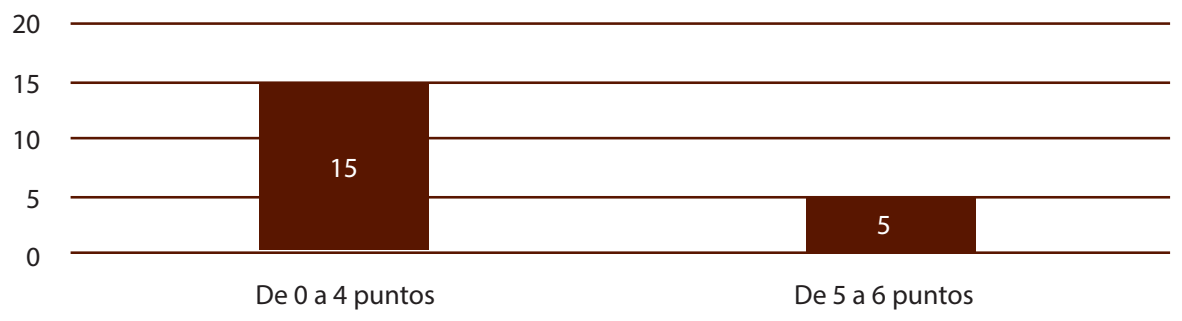

Orientación lateroespacial (Gralifret, 1984).

Puntos logrados por el estudiantado en la instrucción de lateralidad: Diagnóstico.

Figura 4: Resultados del Pretest de orientación lateroespacial (Datos recolectados por la autora con base en Galifret-Granjon (1984).

Durante el trascurso de las sesiones de clase, se pudo evidenciar un avance en las destrezas físicas de los niños y las niñas, a partir del cumplimiento de las tareas psicomotrices ejecutadas. Al concluir la intervención se llevó a cabo la evaluación final con el fin de identificar variaciones en el desempeño del estudiantado de la misma forma en que se aplicó la evaluación inicial.

Final: Coordinación. En cuanto a la coordinación viso-manual, el 95\% del estudiantado realizó el ejercicio en el primer intento, con un número de faltas inferior a 5 y en la coordinación viso-pédica el $100 \%$ logró realizar el ejercicio sin ninguna falta en el primer intento, demostrando avances individuales en el desarrollo de esta habilidad. En las tablas 1 y 2 se detallan los resultados en las áreas de coordinación.

Tabla 1: Comparación pretest y postest de coordinación viso-manual

\begin{tabular}{cccc}
\hline Elementos & Primer intento & 4 & 19 \\
\hline \multirow{2}{*}{ Logro del ejercicio } & Segundo intento & 16 & 1 \\
\hline Cantidad de fallas & 0 a 5 & 1 & 19 \\
& 6 a 10 & 3 & 1 \\
& 11 a 15 & 16 & 0 \\
\hline
\end{tabular}

Nota: Elaboración propia con base en Ozeretski 1988. 
doi: http://dx.doi.org/10.15359/ree.22-1.16

URL: http://www.una.ac.cr/educare

CORREO: educare@una.cr

Tabla 2: Comparación pretest y postest de coordinación viso-pédica

\begin{tabular}{cccc}
\hline Elementos & & Pretest & Postest \\
\hline \multirow{2}{*}{ Logro del ejercicio } & Primer intento & 8 & 20 \\
& Segundo intento & 12 & 0 \\
\hline \multirow{2}{*}{ Cantidad de fallas } & 0 a 5 & 8 & 20 \\
& 6 a 10 & 12 & 0 \\
11 a 15 & 0 & 0 \\
\hline
\end{tabular}

Nota: Datos recolectados por la autora con base en Ozeretski 1988).

En la evaluación previa, el estudiantado tuvo múltiples dificultades para realizar tareas motrices en los ejercicios coordinativos viso-manuales y viso-pédicos, además problemas en la percepción y control corporal esperado para su etapa de desarrollo etario, al igual que en la ubicación del espacio en los diferentes movimientos de dirección; durante la intervención se atendieron estas dificultades, mediante actividades de imitación de movimientos, el circuito motriz y los juegos cooperativos motrices. El estudiantado evaluó el desarrollo de estas habilidades así:

Sí había al principio unos niños que no sabían bien como correr, saltar los conos y esas cosas pero todos los ayudamos y al final ya podían hacer todas las actividades sin problemas, creo que es más fácil hacer los ejercicios cuando los compañeros te ayudan a hacerlo bien.

Final: Control corporal. El 100\% del estudiantado realizó los ejercicios sin fallas y obtuvo el puntaje máximo de la prueba. En la Tabla 3 se integran los resultados iniciales y finales. Se concluye que el estudiantado alcanzó un control corporal acorde con su edad y desarrollo motor, pues superó las dificultades encontradas en el diagnóstico.

Tabla 3: Comparación pretest y postest de control corporal

\begin{tabular}{ccc}
\hline Puntos logrados & Pretest & Postest \\
\hline De 0 a 5 puntos & 4 & 0 \\
De 6 a 8 puntos & 11 & 0 \\
De 9 a 10 puntos & 5 & 20 \\
\hline
\end{tabular}

Nota: Datos recolectados por la autora con base en Berges y Lézine, 1975).

\section{Johanna Lorena Aristizabal-Almanza, Arcelia Ramos-Monobe y Violeta Chirino-Barceló}


Final: Lateralidad. El $100 \%$ del estudiantado realizó exitosamente las instrucciones de lateralidad y ubicación espacial, con ello mostró destreza y el desarrollo de esta habilidad según su etapa de desarrollo, como se muestra en la Tabla 4. Simultáneamente al proceso de desarrollo de las destrezas motoras se pudo observar que el estudiantado logró un mayor grado de atención y concentración que el mostrado al inicio de la intervención, deteniéndose en etapas como la observación y análisis de la situación planteada para emitir una respuesta en equipo, "Siempre estuvimos poniendo cuidado a lo que debíamos hacer", "antes de hacer las cosas pensábamos entre todos como podíamos hacerlo bien"; confirmándose lo propuesto por Vaca (2007). Se observó también que el estudiantado descubrió nuevas formas para realizar las técnicas motrices solicitadas, lo que se complementa con expresiones como: "es mejor realizar el ejercicio así...", se evidenciaron logros en la escucha y el seguimiento de instrucciones desde la interpretación y comprensión de las acciones motrices realizadas.

Tabla 4: Comparación pretest y postest de orientación lateroespacial

\begin{tabular}{ccc}
\hline Puntos logrados & Pretest & Postest \\
\hline De 0 a 4 puntos & 15 & 0 \\
De 5 a 6 puntos & 5 & 20 \\
\hline
\end{tabular}

Nota: Datos recolectados por la autora con base en Galifret-Granjon (1984).

\section{El trabajo en equipo}

En cuanto a la competencia de trabajo en equipo esta investigación se enfocó en la interdependencia positiva desde los procesos de comunicación mediante el diálogo y la escucha; la responsabilidad individual a través del cumplimiento de roles y tareas; el proceso de toma de decisiones desde el análisis, observación y posibles soluciones para emitir acciones colaborativas y por último la cohesión del grupo en el alcance de los objetivos, el compromiso para ayudar y aportar al equipo, en las diferentes actividades programadas dentro de la intervención pedagógica (Johnson et al., 1999).

El diagnóstico de la competencia genérica de trabajo en equipo, se realizó a través de un juego cooperativo, enfocando la interdependencia positiva entendida como ligar mi éxito con el éxito de los demás (Johnson et al., 1999). Ningún equipo cumplió la actividad, las dificultades encontradas fueron: que el proceso de comunicación no fue asertivo; que todos hablaban al mismo tiempo y no mostraban habilidad para escuchar y dialogar ordenadamente. Esto fue evidenciado en comentarios como "Profe es que Mateo no quiere hacer la actividad porque sólo quiere hacer lo que él dice"; "esto está muy difícil y nadie ayuda Miss". Así mismo, en el proceso 
doi: http://dx.doi.org/10.15359/ree.22-1.16

URL: http://www.una.ac.cr/educare

CORREO: educare@una.cr

de toma de decisiones, los niños y niñas no sabían cómo abordar una situación aplicando análisis y observación, lo que mostraba falta de enfoque en sus procesos individuales y escasa cohesión en el equipo; de manera tal que inicialmente se observó que sus experiencias previas se limitaban solo a operar como grupos de trabajo y no como equipos, desorganizados, sin alcanzar la interdependencia positiva.

Al concluir la intervención, se realizó la evaluación final de la competencia. Se pudo observar que el estudiantado asumió una responsabilidad individual en la ejecución de sus roles y al cumplir con sus funciones, apoyando en el proceso a sus pares; también que reconocieron que el éxito personal está ligado al éxito del equipo de trabajo.

En la Tabla 5 se muestran los resultados de la escala de valoración a partir de la autoevaluación realizada por el estudiantado en el proceso de aprendizaje. La percepción sobre el logro de la responsabilidad individual se valoró considerando el cumplimiento de los roles asignados, donde el 95\% del estudiantado manifestó que siempre lo cumplieron. En cuanto al cumplimiento de las tareas asignadas, el $90 \%$ reporta haber cumplido siempre y el $10 \%$ restante algunas veces.

El segundo proceso evaluado fue el de comunicación a través de la escucha de cada integrante del equipo a sus compañeros y compañeras: El $90 \%$ del estudiantado manifestó siempre haberles escuchado y el 10\% restante algunas veces. La toma de decisiones se evaluó a partir de las soluciones propuestas por el estudiantado frente a las dificultades encontradas por el equipo, el $90 \%$ reportó cumplir siempre, mientras que el 10\% restante manifiesta que lo realizó algunas veces.

El proceso de reflexión y cohesión del equipo se midió observando el compromiso para ayudar y aportar todas las habilidades y conocimientos previos del estudiantado para el equipo, el 95\% del estudiantado manifestó que siempre se comprometió, mientras que el 5\% indicó que algunas veces. Por último, se valoraron los procesos de observación, análisis, escucha, y seguimiento de instrucciones ante cada actividad desarrollada en la clase; el $90 \%$ realizó siempre estos procesos antes de la ejecución de los ejercicios; según su autovaloración y que coincide con lo observado durante la intervención.

En cuanto a su percepción en relación con el logro de los ejercicios de lateralidad, coordinación y control corporal, el 90\% del estudiantado manifestó haber alcanzado el objetivo planteado para cada habilidad, mientras que el $10 \%$ restante manifestó que algunas veces, coincidiendo con los resultados obtenidos desde la evaluación inicial y final del test psicomotriz, lo que demuestra, además, su percepción en cuanto a los avances durante su participación activa en el proceso de intervención. 
Tabla 5: Escala de valoración de su ejecución durante el trabajo en equipo en la intervención. Frecuencias de presentación de la tarea en el equipo

\begin{tabular}{|c|c|c|c|}
\hline Tareas esperadas & Siempre & A veces & Nunca \\
\hline Cumplimiento de roles & 19 & 1 & 0 \\
\hline Escucha & 18 & 2 & 0 \\
\hline Propuesta soluciones & 18 & 2 & 0 \\
\hline Compromiso para ayudar y aportar habilidades & 19 & 1 & 0 \\
\hline Cumplimiento de tareas & 18 & 2 & 0 \\
\hline Habilidad de lateralidad & 18 & 2 & 0 \\
\hline Habilidades coordinativas & 19 & 1 & 0 \\
\hline Control corporal & 18 & 2 & 0 \\
\hline Utilización de los sentidos para explorar & 18 & 2 & 0 \\
\hline Procesos de observación, análisis, escucha, y seguimiento de instrucciones & 18 & 2 & 0 \\
\hline
\end{tabular}

La participación activa, reflexiva y propositiva, donde cada estudiante aportó sus aptitudes, saberes y destrezas en pro de la consecución del objetivo (Johnson et al., 1999) favoreció el desarrollo de la competencia de trabajo en equipo, al ligar el éxito del equipo al personal.

A lo largo de las seis sesiones se pudo observar una mejoría progresiva de los niños y niñas respecto a la competencia de trabajo en equipo. Se logró identificar la interdependencia positiva, donde el estudiantado aprendió a escuchar y respetar las opiniones y aportes de su equipo, con el propósito de trabajar en conjunto para lograr diferentes metas desde la contextualización que permitían los juegos y ejercicios lúdicos implementados.

Los tiempos de trabajo otorgados para cada equipo se manejaron conforme a lo establecido en la secuencia didáctica, los niños y niñas realizaron las acciones esperadas dentro de los tiempos indicados para cada actividad. Cabe señalar que, durante la intervención, los equipos de trabajo se conservaron sin modificaciones al igual que los roles, a fin de consolidar su proceso de aprendizaje. Se evidenció un ambiente de comunicación asertiva, de escucha, respeto y colaboración, el cual permitió el éxito del equipo en las tareas desempeñadas donde el estudiantado expresó cosas como: 
doi: http://dx.doi.org/10.15359/ree.22-1.16

URL: http://www.una.ac.cr/educare

CORREO: educare@una.cr

cada uno escuchó a los demás, luego hablamos y nos pusimos de acuerdo...

el líder organizó el equipo y miramos entre todos como podíamos hacer la actividad...

cuando todos hablaban al tiempo, el líder dio turnos para organizarnos...

si cada uno en mi equipo cumplió con su papel y las tareas que les dio la profe, cada uno sabía que tenía que hacer y como tenía que participar para trabajar en equipo.

Se observó que los estudiantes se comprometieron para lograr las metas asignadas y que a través de la asignación de roles cada uno sabía cuáles eran sus funciones (responsabilidad individual) de acuerdo con Gil, Rico y Sánchez-Manzanares (2008), lo que les permitió aportar al grupo desde sus habilidades contribuyendo todas y todos con su mayor esfuerzo para la realización y cumplimiento de cada propósito en equipo "Decidimos entre todos qué papel era el de cada uno y ayudamos al equipo para realizar bien todos los ejercicios".

El proceso de toma de decisiones fue una de las características del trabajo en equipo que más se les dificultó. Mostraron mucha inseguridad para actuar, ya que esperaban una instrucción específica. No obstante, a lo largo de la intervención se pudo ver el avance en cuanto al proceso de análisis de los contextos, lluvia de ideas para abordar la situación y, posteriormente, la emisión de una respuesta (acción colaborativa), lo que permitió un espacio de exploración para el estudiantado (Silberman, 2005).

\section{Aprendizaje activo}

Durante el proceso didáctico se realizó la observación de los elementos asociados a la operacionalización del aprendizaje activo de manera tal que se pudiese determinar si lo esperado al colocar al alumnado como centro del proceso ocurría. Fue así como se puso especial atención en la reflexión individual y grupal en torno a los saberes, la participación, el aprendizaje y la construcción social de conocimiento y motivación; como los indicadores del aprendizaje activo.

Reflexión individual. La reflexión es indispensable dentro del proceso de aprendizaje activo, se provocó el análisis del estudiantado a través preguntas orientadoras que permitieran la activación de sus conocimientos previos (Huber, 2008). Se pudo observar la activación de sus conocimientos en expresiones como: "sé que el trabajo en equipo es trabajar con mi compañero e ir ayudándonos y alcanzar una meta", "si nosotros hicimos algunos ejercicios de correr, saltar y movimientos en clase de educación física la semana pasada ya sabemos cómo hacerlo".

También se observó que la intervención pedagógica facilitó la activación de conocimientos previos del estudiantado para relacionarlas con los nuevos: "Si sabíamos algunas cosas, cuando hicimos la clase había cosas que ya sabíamos de antes, pero también aprendimos muchas otras cosas cuando hicimos las actividades y los juegos".

\footnotetext{
16 Johanna Lorena Aristizabal-Almanza, Arcelia Ramos-Monobe y Violeta Chirino-Barceló
}

Los artículos de la Revista Electrónica Educare del Centro de Investigación y Docencia en Educación de la Universidad Nacional, Costa Rica, se comparten bajo términos de la Licencia Creative Commons: Reconocimiento, № Comercial, Sin Obra Derivada 3.0 Costa Rica. Las autorizaciones adicionales a las aquí delimitadas se pueden obtener en el correo: educare@una.cr 
Reflexionar en forma individual implica la acción de pensamiento que refleja la relación encontrada entre las experiencias vividas y el desempeño alcanzado en el proceso de aprendizaje (Chirino et al., 2015). El estudiantado mostró procesos de reflexión en donde enfocaban sus fuerzas, debilidades y los elementos relevantes del proceso de aprendizaje:

Tuvimos muchas cosas buenas como los papeles que cada uno tenía y también lo que cada uno sabía hacer para ayudar a los demás y hacer las actividades de la clase;

Mmm algunos problemas fueron que unos niños se caían, pero el equipo los ayudo mucho;

también que al principio todos hablaban al tiempo, pero después nos organizamos bien;

algunas cosas las hacíamos mal por el afán, pero luego lo podíamos volver a hacer para mejorar eso.

Se pudo observar que durante la intervención pedagógica el estudiantado analizaba su desempeño, identificaba sus aciertos y desaciertos, se apropiaba de los roles y aportaba todas sus destrezas al equipo de trabajo para conseguir la meta final mediante la interacción con el medio, con el objeto de aprendizaje y entre pares. En cuanto al proceso de aprendizaje expresaron: "lo que debo hacer para trabajar en equipo es saber escuchar, decir soluciones a los problemas, ayudar a mis compañeros, hacer mi mejor esfuerzo para cumplir la meta, y también cumplir con el papel que me pusieron en el equipo". Esto refleja un aprendizaje significativo durante el proceso.

El proceso de reflexión facilitó la integración individual de los saberes previos del estudiantado en contraste con lo que necesitaba saber, descubriendo sus vacíos de información y abriendo canales de aprendizaje desde la motivación (Huber, 2008); también incluyó el análisis conjunto del aprendizaje desarrollado, es decir, la reflexión grupal (Chirino et al., 2015), de manera que se valoró la colaboración para la construcción del conocimiento.

Participación. Implica la acción permanente del estudiantado, apoyada por la observación y la apropiación de su propio desempeño en una actividad (Huber, 2008). Los niños y las niñas fueron conscientes de sus niveles de involucramiento en el proceso de enseñanza-aprendizaje, verbalizándolo de la siguiente manera: "Participamos mucho en la clase..."; "si, todo el tiempo, eso es muy chévere porque hacemos ejercicios, bailamos y hacemos actividades con los amigos"; "todo el tiempo estamos haciendo algo o sea actividades, juegos, ejercicios y bailar". Esto reflejó elementos de tipo emocional en la interacción con sus iguales, lo cual, a la vez, favoreció la motivación.

Se pudo observar que a los niños y niñas se les facilitó el alcance de los objetivos de aprendizaje al integrarse en un equipo de trabajo: "nos iba bien cuando decidíamos hacer las cosas entre todos". La participación dinámica fue una de las características más importantes encontradas durante la realización de las actividades. Para Villardón (2006), la participación del estudiantado se da desde la aplicación de los saberes en su acción, reflexión y cooperación. 
doi: http://dx.doi.org/10.15359/ree.22-1.16

URL: http://www.una.ac.cr/educare

CORREO: educare@una.cr

Aprendizaje social y construcción del conocimiento. Es uno de los elementos principales del aprendizaje activo (Revans, 1983). Durante la intervención, se observó que el aprendizaje social fue importante para que el estudiantado tuviera vivencias que les permitieron desarrollar habilidades y destrezas.

Las metas que debía lograr el estudiantado fueron definidas y explicadas enfocando que el estudiantado conociera la importancia de realizar su máximo esfuerzo para beneficiar el desempeño del equipo. El énfasis radicó en lo que Johnson et al. (1999) manifiestan como interdependencia positiva (ligar mi éxito con el de los demás sujetos). El estudiantado mostró saber lo que se esperaba que lograran en las actividades de aprendizaje, relacionando el desempeño observado con expresiones como: "sí, teníamos claro el objetivo de cada actividad", "si, conocíamos bien cuál era la meta que se debía lograr".

Se observó trabajo conjunto y participativo en la realización de las funciones asignadas (Villasana y Dorrego, 2007). El estudiantado manifestó: "si, miramos antes lo que teníamos que hacer y también quésabía cada uno para ayudar a los compañeros y trabajamos juntos para hacer las cosas bien".

La interdependencia positiva se pudo observar, ya que el estudiantado cumplió sus responsabilidades individuales, respetando el trabajo de las demás personas y apoyando a sus compañeros de equipo para alcanzar las metas, enfatizando en la colaboración desde la aportación de sus habilidades (Johnson et al., 1999).

Finalmente se evidencia el principio de colaboración (Torrelles et al., 2011), donde este se $\mathrm{da}$, en la medida en que el estudiantado tenga claros los objetivos de aprendizaje y las aptitudes necesarias para que el grupo funcione efectivamente desde la interdependencia positiva.

Motivación. Se refiere a la preparación y disposición mental, para la ejecución de una acción con interés y diligencia (Villa y Poblete, 2007). Se observó la participación e interés al trabajar cooperativamente el estudiantado, lo que facilitó la comprensión de las tareas a realizar, el desarrollo de habilidades motoras y de los elementos observados del trabajo en equipo apoyándose mutuamente en la interacción.

Se observaron también expresiones que reflejan la existencia de interdependencia positiva, donde el estudiantado manifestó agrado hacia el desarrollo de las actividades:

Es divertido porque uno se ayuda con los compañeros y porque así puede lograr hacer cosas

Me gusta estar con los amigos y ayudar cuando tenemos problemas

es muy bueno ver que todo lo hacemos unidos y que todos compartimos para lograr llegar a hacer algo. 
La motivación es importante para el aprendizaje activo, ya que es un detonante del proceso de activación de los aprendizajes previos (Chirino et al., 2015). La motivación se pudo observar cuando el estudiantado expresó apreciaciones como:

Profe ya vamos a hacer los equipos, a mí me gusta trabajar con mis compañeros, es muy divertido en otras clases hacemos equipos y sabemos cómo se hace", "es genial trabajar con los compañeros, ayudarnos, y trabajar en equipo, si, en Educación Física hacemos actividades parecidas a estas donde corremos, saltamos y esos ejercicios.

La cohesión de los equipos se pudo observar en el fortalecimiento de las relaciones interpersonales, al comparar el inicio y el final del curso, los niños y las niñas se mostraron con motivación por el aprendizaje, se comunicaron con mayor facilidad, solucionaron dificultades, incluso sin la intervención docente. Schwartz y Pollishuke (1998) declaran que la motivación se logra al crear un ambiente de aprendizaje cálido, acogedor y seguro, con el fin de propiciar el desarrollo personal del estudiantado a través de su interacción y conexión en un equipo de trabajo.

A través de las estrategias de aprendizaje activo se provocó la reflexión individual y grupal para el aprendizaje de habilidades psicomotrices e, implícitamente, de la competencia de trabajo en equipo. Se observó que el estudiantado se interesó por aprender sobre todo al participar en colectivo en actividades con enfoque lúdico, implementadas con base en la planeación de la intervención pedagógica.

\section{Conclusiones}

La intervención pedagógica implementada con un enfoque de aprendizaje activo apoyó el desarrollo de la expresión corporal enfatizada en los procesos psicomotores de lateralidad, coordinación y control corporal de los niños y niñas de primer grado, incentivó el aprendizaje a través del movimiento y la exploración en la interacción con sus compañeros, compañeras y su ambiente.

Se identificó también que esta intervención pedagógica permitió detectar, reforzar y superar múltiples dificultades motrices del estudiantado; quienes adquirieron diversas habilidades y destrezas acordes con su proceso de desarrollo corporal, involucrando diferentes procesos mentales como el análisis y la observación basada en la reflexión de sus vivencias.

El estudiantado mostró la interdependencia positiva al reconocer y respetar a la otra persona y considerar sus aportes individuales al equipo. Mejoraron significativamente en sus procesos de comunicación, escucha, análisis y toma de decisiones de acuerdo con su edad y a partir del cumplimiento de roles y responsabilidades y la ayuda a sus pares para lograr los objetivos trazados en cada sesión de clase.

En cuanto a la experiencia, se reconoce la importancia de formarse como educador o educadora con disposición por innovar, por replantear su quehacer, con el fin de apostarle realmente a una concepción constructivista, dinámica y creativa como lo es el aprendizaje activo. 
doi: http://dx.doi.org/10.15359/ree.22-1.16

URL: http://www.una.ac.cr/educare

CORREO: educare@una.cr

Así, entonces, los principales resultados arrojados tras el proceso de intervención determinaron que la didáctica colaborativa a la luz del aprendizaje activo favoreció el desempeño del estudiantado en el nivel psicomotor, específicamente, en sus habilidades coordinativas, de lateralidad y control corporal yel de logro de la competencia de trabajo en equipo; facilitóla construcción del conocimiento desde la conexión de los aprendizajes previos con los nuevos; y la reflexión sobre aciertos, desaciertos y participación en un equipo de trabajo desarrolló autonomía y responsabilidad.

No obstante, se considera que esta investigación sobre la intervención pedagógica fue realizada en un lapso corto de tiempo por lo que implica que estos resultados reflejan un análisis en un contexto determinado y no pueden ser generalizados, sólo utilizados como referentes. Se estima que una intervención prolongada puede conducir aún más a resultados confirmatorios de lo que aquí fue encontrado. Por tanto, en el sentido de la acción docente se recomienda plantear esfuerzos sistemáticos de intervención continua con un énfasis en la reflexión de la práctica en el aula para continuar el ciclo de la investigación acción.

Como parte de un proceso de investigación se recomienda su aplicación en otros contextos disciplinares con el fin de ampliar los procesos de investigación y, con ello, nuevos resultados para este grupo de edad. Así como se considera conveniente ampliar el rango de edad o de instituciones; de manera que al intervenir se aumente el número de docentes que se sensibilicen frente al aprendizaje activo como una metodología que mejora sus prácticas en el aula. Así, pues, los cuerpos docentes podrán realizar innovaciones educativas en su contexto, teniendo en cuenta metodologías que colocan al alumnado como el centro del proceso.

\section{Referencias}

Berges, J. y Lézine, I. (1975). Test de imitación de gestos. Barcelona: Científico Médica.

Blanco, P. (2006). El rompecabezas cooperativo para adquirir competencias de desarrollo personal y social en educación física. Revista Iberoamericana de Educación, 39(1), 1-8. Recuperado de http://rieoei.org/1451.htm

Bolaños, G. (2006). Educación por medio del movimiento y expresión corporal (11 a reimp.). San José, Costa Rica: Euned.

Carr, W. y Kemmis, S. (1988). Teoría crítica de la enseñanza: La investigación-acción en la formación del profesorado. Barcelona: Martínez Roca.

Chirino, V., Ramos, A. y Lozano, A. (2015). Reenfocando el aprendizaje activo hacia un modelo de aula invertida. Pautas para la acción docente. CIIE. Revista del Congreso Internacional de Innovación Educativa, 1(1), 164-174. Recuperado de https://drive.google.com/file/ d/OBxu5PCww2WDQQzBhQjY2bUV1UDQ/view 
Cisterna, F. (2005). Categorización y triangulación como procesos de validación del conocimiento en investigación cualitativa. Theoria, 14(1), 61-71. Recuperado de http://www.ubiobio.cl/ theoria/v/v14/a6.pdf

Da Fonseca, V. (2000). Estudio y génesis de la psicomotricidad. Barcelona: Inde.

Domingo, J. (2008). El aprendizaje cooperativo. Cuadernos de Trabajo Social, 21, 231-246. Recuperado de https://dialnet.unirioja.es/ejemplar/203659

Elliott, J. (1993). El cambio educativo desde la investigación-acción. Madrid: Morata.

Galifret-Granjon, N. (1984). Batería Piaget-Head. En R. Zazzo (Autor), Manual para el examen psicológico del niño (pp. 91-152). Madrid: Narcea.

García, J. A. (2009). Psicomotricidad y educación infantil. Revista Innovación y Experiencias Educativas, 16, 1-10.

Gil, P., Gutiérrez, E. C. y Madrid, P. D. (2013). Incremento de las habilidades sociales a través de la expresión corporal: La experiencia en clases de iniciación al baile. Cuadernos de Psicología del Deporte, 12(2), 83-88. Recuperado de http://revistas.um.es/cpd/article/ view/177821/149501

Gil, F., Rico, R. y Sánchez-Manzanares, M. (2008). Eficacia de equipos de trabajo. Papeles del Psicólogo, 29(1), 25-31. Recuperado de http://www.redalyc.org/articulo.oa?id=77829104

Gómez, R. H. (2002). La enseñanza de la educación física en el nivel inicial y el primer ciclo de la EGB: Una didáctica de la disponibilidad corporal. Buenos Aires: Stadium.

Hernández, J. y Anello, E. (1998). Trabajo en equipo. Red Nacional de Formación y Capacitación Docente. Quito: MEC.

Huber, G.L. (2008). Aprendizajeactivoy metodologías educativas. Revista deEducación, extraordinario, 59-81. Recuperado de http://www.revistaeducacion.mec.es/re2008/re2008 04.pdf

Johnson, D. W., Johnson, R. T. y Holubec, E. J. (1999). El aprendizaje cooperativo en el aula. Barcelona: Paidós.

Koo, L. C. (1999). Learning action learning. Journal of Workplace Learning, 11(3), 89-94. doi: https://doi.org/10.1108/13665629910264244

Lewin, K. (1988). Acción-investigación y problemas de las minorías. Revista de Psicología social, 3(2), 229-240. doi: https://doi.org/10.1080/02134748.1988.10821586

Osorio, K.y López, A. (2014). La retroalimentación formativa en el proceso de enseñanza-aprendizaje de estudiantes en edad preescolar. Revista Iberoamericana de Evaluación Educativa, 7(1), 1330. Recuperado de https://revistas.uam.es/index.php/riee/article/view/3383 
doi: http://dx.doi.org/10.15359/ree.22-1.16

URL: http://www.una.ac.cr/educare

CORREO: educare@una.cr

Ozeretski, N. A. (1988). Test motores de Ozeretski. Manual de diagnóstico psicológico. Madrid: Mepsa.

Revans, R. W. (1983). Action learning: Its terms and character. Management Decision, 21(1), 39-50. doi: https://doi.org/10.1108/eb001310

Schwartz, S. y Pollishuke, M. (1998). Aprendizaje activo: Una organización de la clase centrada en el alumnado ( $2^{\mathrm{a}}$ ed.). Madrid: Narcea.

Silberman, M. (2005). Aprendizaje activo: 101 estrategias para enseñar cualquier tema. México: Pax. Recuperado de https://josedominguezblog.files.wordpress.com/2015/06/aprendazajeactivo-101-estrategias-para-ensenar-1.pdf

Torrelles, C., Coiduras, J., Isus, S., Carrera, F. X., París, G. y Cela, J. M. (2011). Competencia de trabajo en equipo: Definición y categorización. Profesorado: Revista de currículum y formación del profesorado, 15(3), 329-344. Recuperado de https://recyt.fecyt.es/index.php/profesorado/ article/view/41484/23585

Touriñan, J. M. (2011). Intervención educativa, intervención pedagógica y educación: La mirada pedagógica. Revista Portuguesa de Pedagogía, 283-307. Recuperado de http:// files.secu304tv-com.webnode.com.co/200000235-a21f9a316a/intervenciu00F3n\%20 pedagu00F3gica.pdf

Vaca, M. J. (2007). Un proyecto para una escuela con cuerpo y en movimiento. Ágora para la Educación Física y el Deporte, 4-5, 91-110. Recuperado de https://www5.uva.es/agora/ revista/4/agora4-5 vaca $5 . p d f$

Valenzuela, J. R. y Flores, M. (2012). Fundamentos de la investigación educativa (Vol. 2). México: Trillas.

Villa, A. y Poblete, M. (2007).Aprendizaje basado en competencias. Una propuesta para la evaluación de las competencias genéricas. Bilbao: Mensajero. Recuperado de https://es.slideshare.net/ andri arg/aprendizaje-basado-en-competencias-24974231

Villardón, L. (2006). Evaluación del aprendizaje para promover el desarrollo de competencias. Educatio Siglo XXI, 24, 57-76. Recuperado de http://revistas.um.es/educatio/article/ view/153/136

Villasana, N. y Dorrego, E. (2007). Habilidades sociales en entornos virtuales de trabajo colaborativo. RIED: Revista Iberoamericana de Educación a Distancia, 10(2), 45-74. doi: https://doi.org/10.5944/ried.2.10.993 

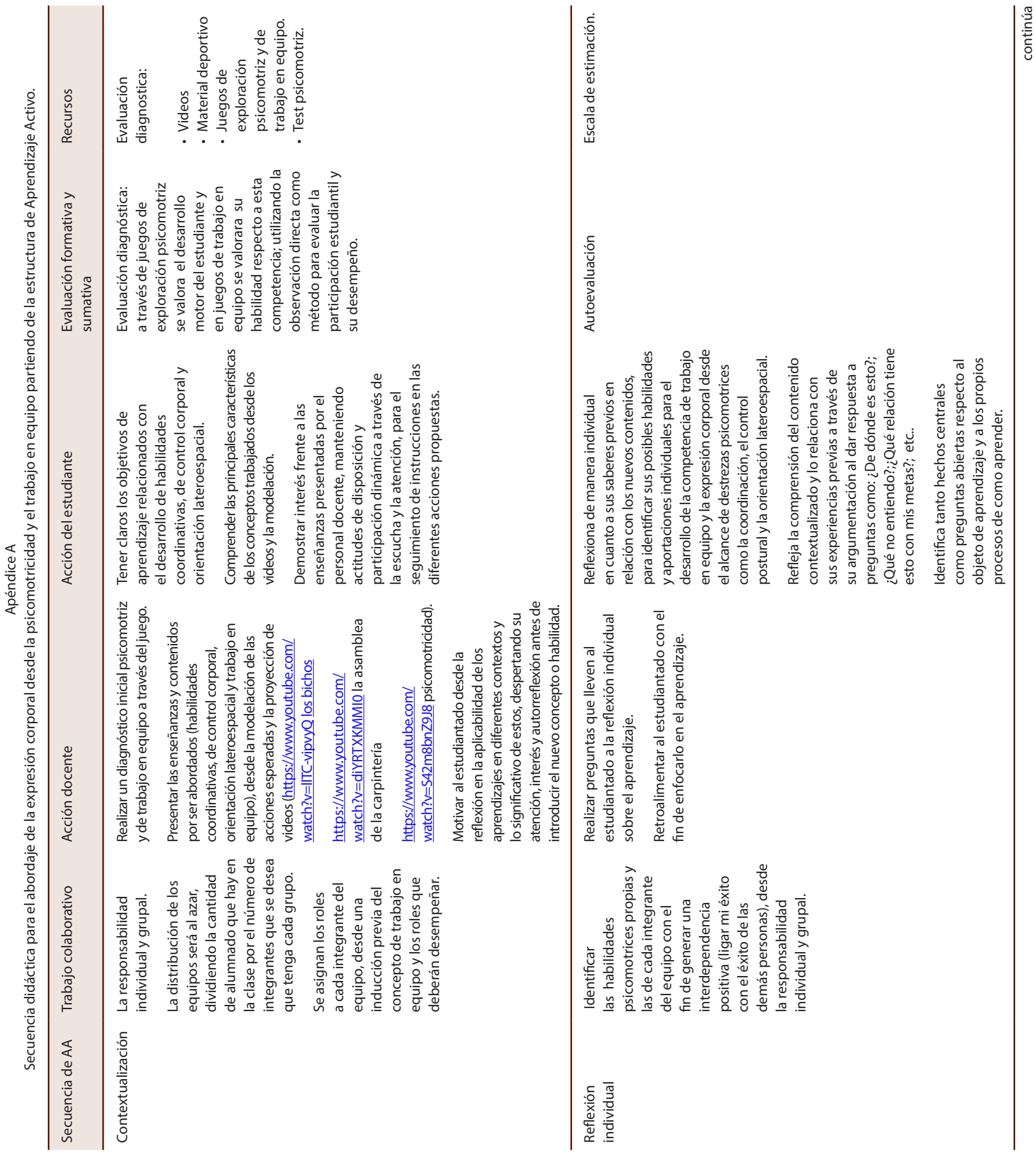
doi: http://dx.doi.org/10.15359/ree.22-1.16

URL: http://www.una.ac.cr/educare

CORREO: educare@una.cr

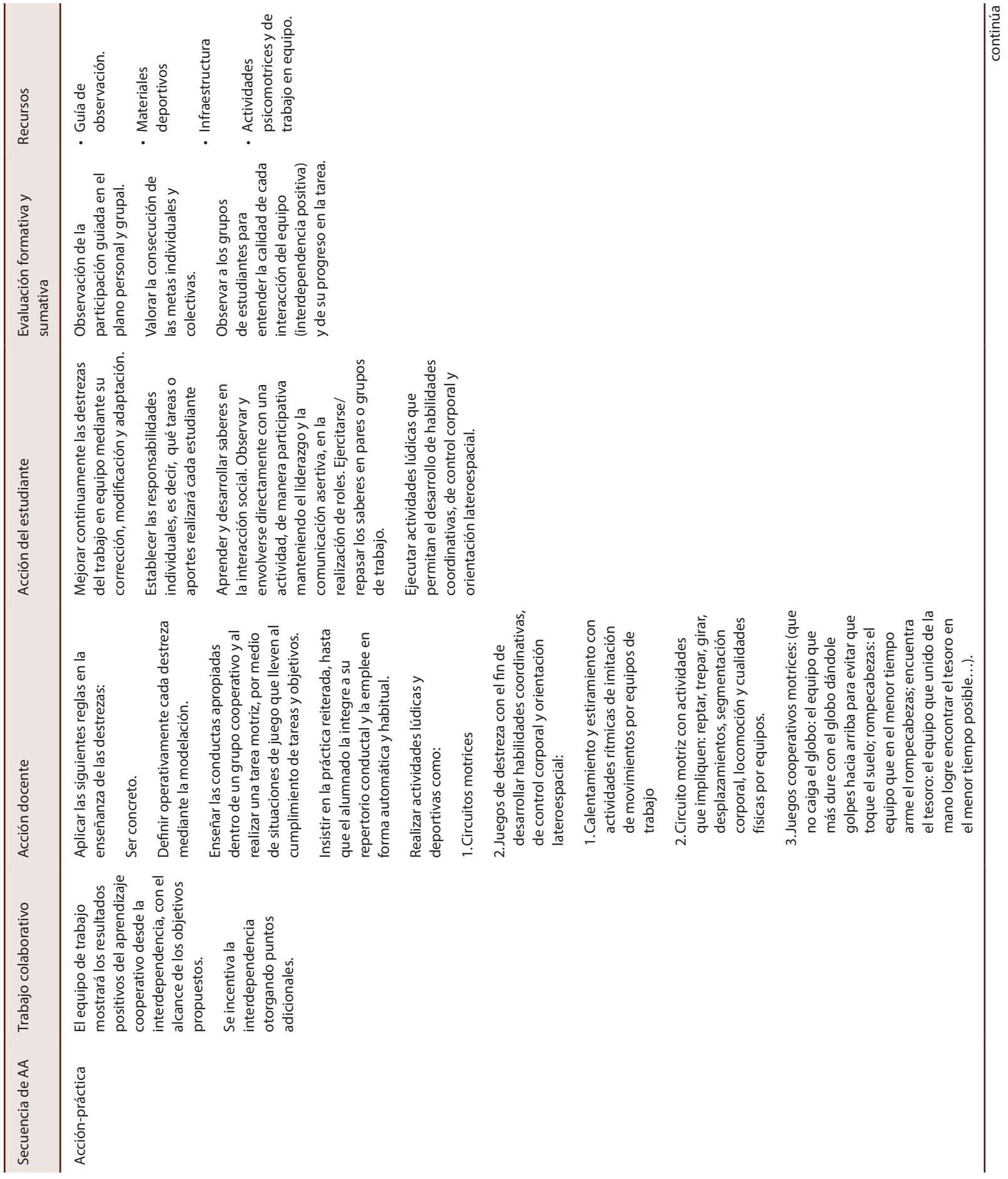

24 Johanna Lorena Aristizabal-Almanza, Arcelia Ramos-Monobe y Violeta Chirino-Barceló 


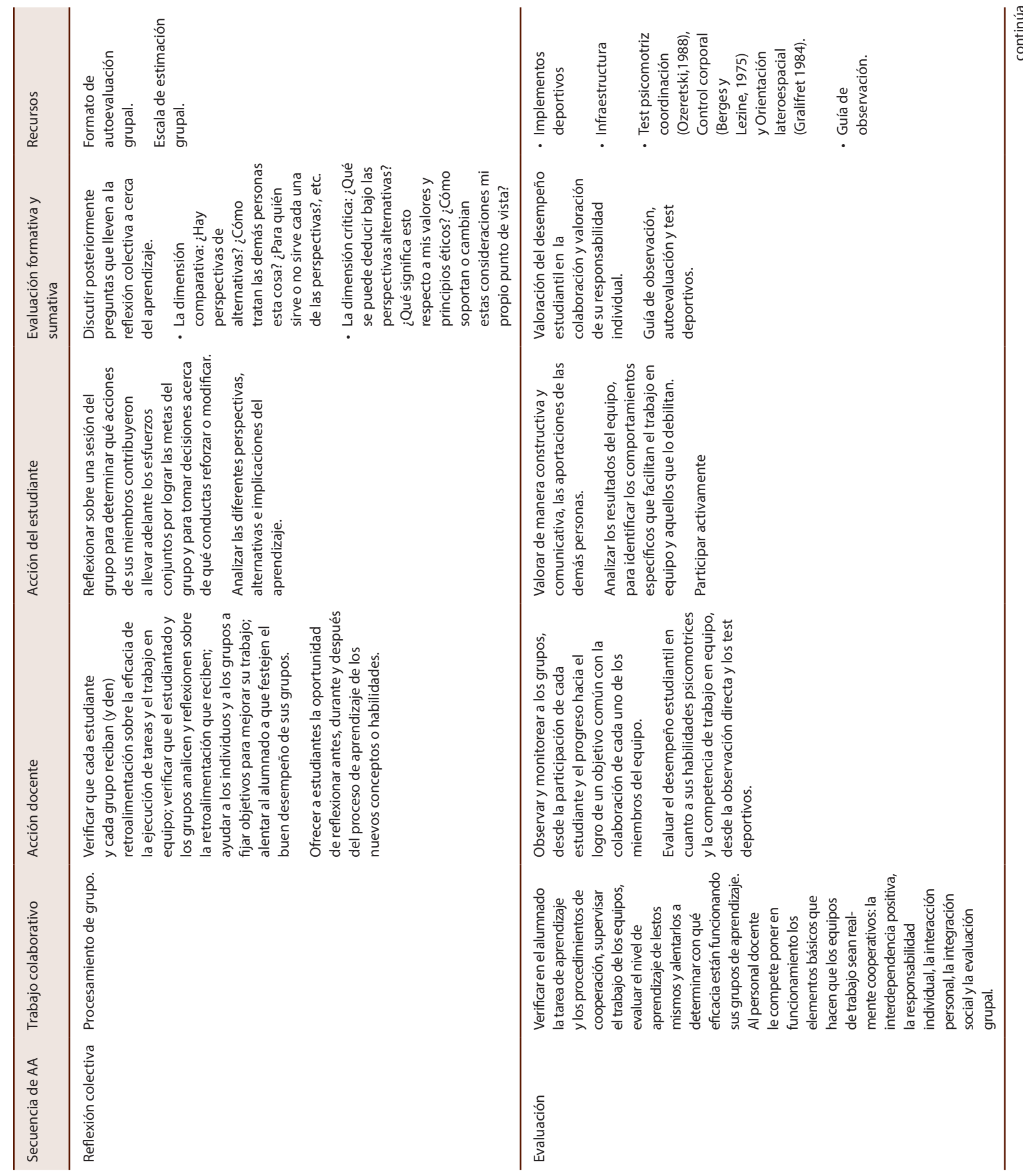


doi: http://dx.doi.org/10.15359/ree.22-1.16

URL: http://www.una.ac.cr/educare

CORREO: educare@una.cr

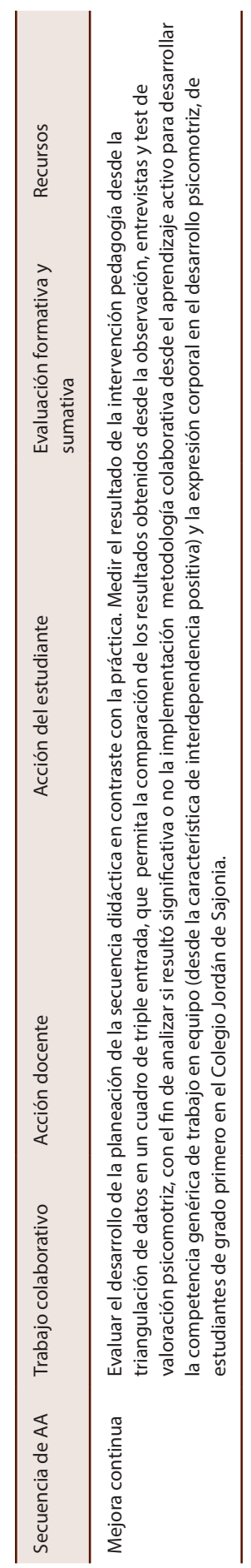

